\title{
ANÁLISIS TAXONÓMICO DE LAS ESPECIES DE PLANTAS INTRODUCIDAS EN COSTA RICA
}

\author{
Eduardo Chacón ${ }^{1}$ y Guido SABorío-R. ${ }^{2}$ \\ Escuela de Biología, Universidad de Costa Rica, 2060 San José, Costa Rica \\ 1edchacon@gmail.com - 29saborio@gmail.com
}

\begin{abstract}
Introduced plants have become an important component of vegetation around the world. Both data bases and lists of introduced species allow us to compare their distribution and effects on natural ecosystems in different regions, giving us a better understanding of their ecology to develop preventive strategies. Here we present a taxonomic analysis of 1048 introduced plant species from Costa Rica, included in 609 genera of 148 families. We consider that the number of introduced plants is underestimated and it may increase as more investigation is developed in this field. The family with the largest number of species is Poaceae (118 spp.) followed by Fabaceae, with a total of 74. Moreover, 116 families have less than 10 species. A high percentage of the introduced species are herbs (55\%) and the use of the introduced plants in Costa Rica is mainly ornamental (52\%). For multiple purposes is used $8.9 \%$ of the species, as ornamental, medicinal, and edible. Populations status of the introduced plants in Costa Rica is poorly known for most species and for about $31 \%$ of the species analyzed here this information is completely unknown. From the rest of species, $47 \%$ are cultivated and $22 \%$ naturalized. The geographical origin of these species is extremely diverse; however, tropical Asia (19.4\%) and South America (18.6 \%) are the most common origins of introduced plant species in Costa Rica. Here we present the first research on introduced plant species in Costa Rica and to our knowledge the first taxonomic analysis of the introduced flora in the Neotropics. To see the resulting catalogue of introduced plants in Costa Rica check http://i3n.iabin.net (whole address at the end of Bibliografía consultada).
\end{abstract}

Resumen. Las plantas introducidas se han convertido en un componente importante de la vegetación de casi todos los países del mundo. Tanto las bases de datos como listas de especies introducidas nos permiten comparar su distribución y sus efectos en los ecosistemas naturales en diferentes regiones, dándonos un mejor conocimiento de su ecología para desarrollar estrategias preventivas. Aquí presentamos el análisis de 1048 especies de plantas introducidas en Costa Rica, pertenecientes a 609 géneros y 148 familias. Consideramos que este número de especies introducidas es una subestimación, que debe aumentar cuando se genere más investigación sobre el tema. La familia con el mayor número de especies introducidas es Poaceae (118 spp.), seguida por Fabaceae con 74. Del total, 116 familias aportan 10 o menos especies. El $55 \%$ de las especies introducidas son hierbas. El uso más común que se les da a las especies de plantas introducidas en Costa Rica es ornamental (52\%). Un $8.9 \%$ de las especies tienen varios usos, como ornamentales, medicinales y comestibles. En Costa Rica es escasa o nula la información sobre el estado poblacional de las especies introducidas; no existen datos del 31\% de las especies aquí registradas. El resto son especies cultivadas ( $47 \%)$ o naturalizadas $(22 \%)$. El origen geográfico de estas especies es muy variado; sin embargo, Asia tropical (19.4 \%) y Sudamérica (18.6\%) son las regiones de origen del mayor número de especies introducidas en Costa Rica. Aquí presentamos el primer estudio de las plantas introducidas en Costa Rica y, a nuestro conocimiento, el primer análisis taxonómico de la flora introducida en la región neotropical. El catálogo resultante de las plantas introducidas en Costa Rica se hallará en http://i3n.iabin.net (ver dirección completa al final de Bibliografía consultada).

PALABRAS Clave / KeY words: Plantas introducidas / Introduced plants, vegetación / vegetation, Costa Rica, región neotropical / Neotropics

Las plantas introducidas se han convertido en un componente importante de la vegetación de casi todos los países del mundo (Weber 1997). Algunas de esas especies nos ofrecen muchos beneficios; sin embargo, el incremento en la naturalización y expansión de algunas especies produce un serio impacto ecológico (Parker et al. 1999, D'Antonio \& Vitousek 1992, Higgins et al. 1999, Kollman \& Bañuelos 2004). Después de la destrucción de hábitats, la introducción de especies es la segunda causa de extinción y de 
amenaza para las especies nativas en los continentes y es la primera causa de extinción y amenaza para especies nativas en islas oceánicas (Mauchamp 1997, Daehler et al. 2004).

Debido a su gran importancia, las especies introducidas han sido objeto de numerosos estudios en las últimas décadas. Esto ha generado mucha información sobre aspectos ecológicos de las invasiones, así como características clave de las especies invasoras y de las comunidades invadidas (Reichard \& Hamilton 1997, Weber 1997). Muchos de estos estudios se han enfocado, por ejemplo, en evaluar la hipótesis de la resistencia biótica propuesta por Elton (1958). Esta hipótesis señala que las comunidades ricas en especies tienen una mayor resistencia a la invasión de especies que las comunidades con pocas especies. Sin embargo, los resultados obtenidos en diferentes estudios son contradictorios (Maron \& Vila 2001, Espinosa-García et al. 2004).

Otros estudios han tratado de determinar qué características hacen a una especie más propensa a ser invasora (Baker 1967, Rejmanek \& Richardson 1996, Reichard \& Hamilton 1997, Rambuda \& Johnson 2004). Algunos autores proponen que las plantas autocompatibles son mejores colonizadoras que las autoincompatibles (Baker 1967, Rambuda \& Johnson 2004). Rejmanek \& Richardson (1996) observaron que estadios juveniles de corta duración y períodos cortos entre grandes producciones de semillas son dos características comunes de las especies de Pinus cultivadas que son más invasoras. Sin embargo, estos estudios se han enfocado en un área en particular (Rambuda \& Johnson 2004) o en un grupo taxonómico (Rejmanek \& Richardson 1996), lo cual hace difícil generalizar los resultados.

Se ha generado un número importante de bases de datos y listas de especies de plantas introducidas en varios países del mundo (Vitousek et al. 1996, Weber 1997, Myers \& Bazely 2003, Pyšek et al. 2003, Kühn 2004). Esto permite, con algunas limitaciones, comparar el comportamiento de las especies en distintos lugares, lo cual es sumamente importante para poder entender mejor la ecología de las plantas invasoras y, así, desarrollar estrategias preventivas (Pauchard et al. 2004). Reichard y Hamilton (1997), con base en un análisis realizado con plantas leñosas introducidas en Norteamérica, recomiendan no permitir la introducción de plantas en un determinado país si se sabe que éstas han causado serios daños en otros lugares.

A pesar de la gran cantidad de información que se ha generado, la mayoría se ha recopilado en zonas templadas, por lo que casi no existe información de este tipo en los trópicos. Los ecosistemas tropicales tienen características muy distintas a las de ecosistemas de zonas templadas; por tanto, el comportamiento de las plantas exóticas podría ser muy diferente. De allí la importancia de generar información sobre las plantas introducidas en los países de zonas tropicales. Costa Rica no es la excepción y la información que existe sobre las plantas introducidas es casi nula. En un estudio preliminar, Bernhardt \& Koch (1994) enumeran 27 especies de plantas europeas introducidas en zonas montañosas de Costa Rica. El Instituto Nacional de Biodiversidad (INBio, www.inbio.ac.cr/) calcula que en el país existen unas 1500 especies de plantas introducidas, de las cuales esta organización presenta una lista de 62 especies. Aparte de estas dos referencias específicas sobre plantas introducidas, los textos sobre flora de Costa Rica mencionan pocas especies introducidas. Es importante destacar la falta casi total de información sobre ecología de las especies de plantas introducidas en Costa Rica, a pesar de que algunas de ellas se han extendido incluso dentro de algunos parques nacionales.

El objetivo de este trabajo es presentar (en Internet) la primera lista de especies de plantas introducidas en Costa Rica, con información sobre hábito, uso, origen y posible estado poblacional de la especie en este país. Debido a la falta de información que existe sobre el tema y a que continuamente se introducen nuevas especies de plantas en el país, no es posible elaborar una lista completa de especies introducidas en Costa Rica. Además, el estado poblacional de las especies presentadas aquí puede estar sujeto a cambios en el futuro. Consideramos que es importante dar a conocer esta información, porque puede impulsar la investigación de las plantas y de otros organismos introducidos en Costa Rica y, en general, en la región neotropical.

\section{Materiales y Métodos}

Para recopilar la información se utilizó literatura disponible sobre la flora de Costa Rica, la base de datos y los especímenes del Herbario de la Universidad de Costa Rica (USJ), una lista preliminar del Jardín Botánico Lankester (C.O. Morales, datos sin publicar) y el Herbario Nacional de Costa Rica (CR). Esta lista no es definitiva, ya que subestima numerosas especies introducidas, de familias tales como Orchidaceae y Cactaceae, que se encuentran en jardines privados. Además, no se tiene información sobre helechos (Pteridophyta), especies que actualmente se importan, 
ni sobre las plantas del Jardín Botánico Wilson.

Cada especie se catalogó según familia, autor, nombre común en español, hábito, uso y lugar de origen. La familia a la que cada especie pertenece se asignó con base en la clasificación del Missouri Botanical Garden en la base de datos TROPICOS. Para evitar sinónimos, los nombres de las especies se compararon con el International Plant Name Index (http://www.ipni.org), de donde también se tomaron los autores para el nombre de cada especie. Los nombres comunes, el hábito y el uso se tomaron de la misma fuente de origen de cada especie, por conocimiento previo, consulta a especialistas o por datos en los especímenes de los herbarios. Algunos datos de origen se obtuvieron en la página web del Germplasm Resources Information Network, del Departamento de Agricultura de los Estados Unidos (http://www.ars-grin. gov) o del Electronic Plant Information Center (http:// www.rbgkew.org.uk/epic/index.htm). Los lugares de origen se asignaron según las unidades geográficas de Brummitt (2001, Fig. 1), que dividen el mundo en nueve regiones, ignorando límites políticos: Europa, África, Asia templada, Asia tropical, Australasia, Pacífico, Norteamérica, Sudamérica y Antártida. Para el análisis, las especies cuyo ámbito de distribución natural abarca más de una de estas regiones se asignaron a una categoría denominada "varios".

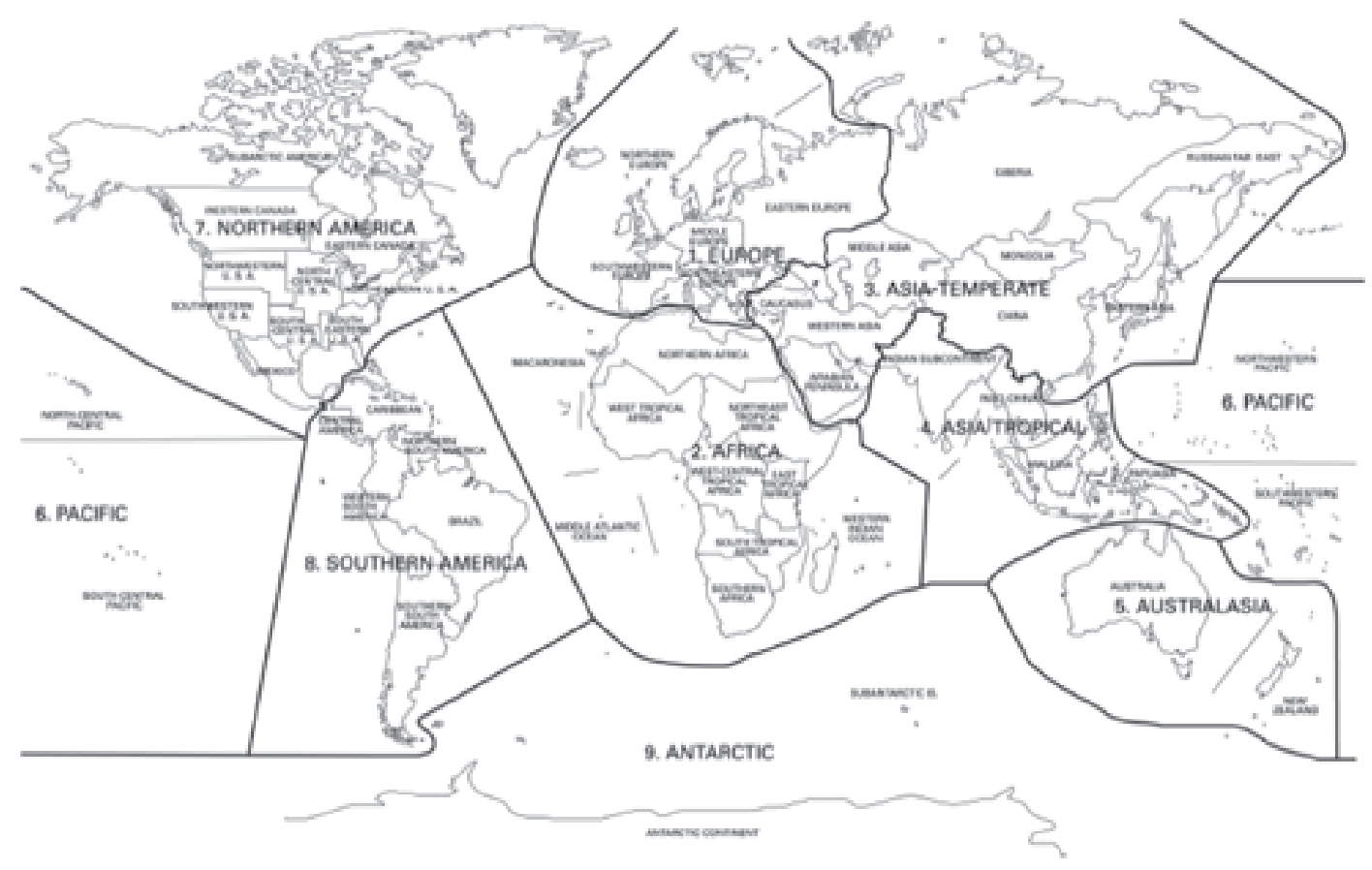

Fig. 1. División geográfica utilizada para estandarizar el origen de las plantas introducidas (Tomado de Brummitt 2001).

\section{Resultados}

El análisis presenta un total de 1048 especies de plantas introducidas en Costa Rica, las cuales pertenecen a 609 géneros de 148 familias. Puesto que el catálogo resultante es muy extenso, no es posible incluirlo aquí, pero podrá consultarse en la página web de la Red Interamericana de Información sobre Biodiversidad (IABIN, http://i3n.iabin.net, ver dirección completa al final de Bibliografia consultada). La familia con el mayor número de especies introducidas es Poaceae
(118 spp.), seguida por Fabaceae con 74 (Cuadro 1). Del total de familias, 116 tienen 10 o menos especies introducidas (Fig. 2). Un alto porcentaje de las especies introducidas son hierbas (Fig. 3) y, en general, el uso más común que se da a las plantas introducidas es ornamental (Fig. 4). Aunque en Costa Rica la información sobre el estado poblacional de las especies introducidas es escasa o nula, la mayoría de las especies aquí presentadas se encuentran cultivadas 
(Fig. 5). Finalmente, Asia tropical y Sudamérica son las regiones de origen del mayor número de especies introducidas en Costa Rica (Fig. 6).

\section{DISCUSIÓN}

Las 1048 especies de plantas introducidas en Costa Rica, registradas aquí, representan casi el $10 \%$ de la flora nacional. Es importante aclarar que esta lista incluye varias especies que no han sido consideradas como parte de la flora nacional en los tratados de la flora costarricense. Aunque hay muy pocos estudios en los trópicos para realizar comparaciones, este valor es similar al comunicado para Chile (15\%), Puerto Rico (12\%) y Australia (11\%), pero es mucho menor que el divulgado para Nueva Zelanda (50\%) (Myers \& Bazely 2003).

El gran número de especies en las familias Poaceae, Fabaceae y Asteraceae es, en parte, reflejo del gran número de especies que tienen estas familias en el mundo (Cuadro 1). Sin embargo, la familia Poaceae tiene casi el doble de especies que las familias siguientes. Este resultado es consistente con otros análisis de flora introducida (Daehler 1998, Pyšek 1998). Muchas especies de gramíneas han sido introducidas intencionalmente en diversas regiones del mundo, ya que a esta familia pertenecen importantes cultivos, como arroz, maíz, trigo, cebada, caña de azúcar y muchas especies utilizadas como forrajes. Además, muchas especies de esta familia han sido introducidas accidentalmente junto con especies de importancia económica.

Las Poáceas o Gramíneas poseen características que les confieren un alto potencial como especies invasoras, ya que se propagan rápidamente por crecimiento vegetativo, por lo que alcanzan altas densidades, su polinización es abiótica, producen un gran número de semillas pequeñas, que se dispersan fácilmente, tienen ciclo de vida anual y fotosíntesis favorecida en áreas abiertas expuestas al sol (Daehler 1998, Myers \& Bazely 2003). Además, algunas especies de esta familia tiene una densa producción de paja (Daehler 1998) y alteran los regímenes naturales de fuego, lo que favorece su propio reestablecimiento (D'Antonio $\&$ Vitousek 1992), como sucede en el Pacífico norte de Costa Rica con el pasto jaragua (Hyparrhenia rufa), nativo de África.

A Poaceae pertenecen muchas especies invasoras, que en Costa Rica son de importancia económica por ser consideradas malezas de varios cultivos. Por ejemplo, los pastos jaragua (H. rufa), kikuyo (Pennisetum clandestinum), pasto de elefante
(Pennisetum purpureum), estrella africana (Cynodon nlemfuensis) y calinguero (Melinis minutiflora), que fueron intencionalmente introducidos para forraje de ganado, pero se han convertido en un problema que debe tomarse en cuenta en planes de conservación en algunos sitios.

En el caso de la familia Fabaceae (en sentido amplio), la segunda en importancia, el alto número de especies se debe a que muchas fueron introducidas por su importancia económica para consumo humano, como forraje para ganado o como cobertura del suelo y fijadoras de nitrógeno. Varias especies de esta familia fueron introducidas accidentalmente junto con semillas de pastos, como es el caso de Ulex europaeus, arbusto común en el Parque Nacional Volcán Poás y en otras partes altas de la Cordillera Volcánica Central, y de varias especies naturalizadas del género Trifolium. Una característica de esta familia, que aumenta su potencial como invasora, es la capacidad de fijar nitrógeno en el suelo, pues esto aumenta la probabilidad de que una especie introducida sobreviva en un ambiente pobre en nitrógeno (Daehler 1998). En la familia Asteraceae, la tercera en importancia por el número de especies, muchas han sido introducidas en Costa Rica como plantas ornamentales y medicinales. Sin embargo, el número de especies introducidas parece estar en función del gran número total de especies que tiene esta familia (Cuadro 2), lo que concuerda con otros estudios (Daehler 1998, Pyšek 1998).

El alto número de especies en las familias Cactaceae y Arecaceae (Cuadro 2) se debe principalmente a que las especies de estas familias son muy utilizadas como ornamentales. Sin embargo, hay que considerar que varias especies de la familia Cactaceae han sido invasoras en otros países, v.g. Australia, donde fue necesario aplicar medidas de control biológico para reducir las poblaciones (Myers \& Bazely 2003).

Algunas familias, como Orchidaceae y Rubiaceae, a pesar de tener muchas especies en el mundo ( $c a$. 24000 y 10 200, respectivamente, Mabberley 1997), tienen relativamente pocas especies introducidas en Costa Rica (8 y 17 especies, respectivamente), resultado similar al obtenido en otros estudios (Daehler 1998, Pyšek 1998). Pocas especies de estas familias están naturalizadas y, en general, no representan una amenaza de convertirse en invasoras. Como mencionamos anteriormente, el número de especies introducidas de la familia Orchidaceae está subestimado, debido a que muchas especies son adquiridas ilegalmente por coleccionistas y no se tienen registros de ellas. Muchas de estas introducciones suelen ser efímeras y sólo quedan en colecciones privadas. Una excepción es la 
orquídea terrestre africana Oeceoclades maculata, que tiene poblaciones naturalizadas en Costa Rica y ha sido una colonizadora muy agresiva en otros países (Dressler 2003). En Rubiaceae, Coffea arabica (café) en la Isla del Coco y C. canephora en la Estación Biológica La Selva (Sarapiquí, Heredia) son consideradas invasoras (P. Madriz y O. Vargas, com. pers.).

La mayoría de las especies introducidas, registradas aquí, son hierbas (Fig. 3). Este resultado en parte se debe a que este es el hábito más común de las plantas y es, además, el hábito más común en las familias con más especies introducidas (Poaceae, Fabaceae, Asteraceae). Muchas plantas introducidas como ornamentales, comestibles y medicinales tienen este tipo de hábito. En el caso de los árboles, varias especies han sido introducidas como ornamentales y otras especies como maderables.

Consistente con otros estudios, el uso más común de las plantas introducidas en Costa Rica es ornamental (Fig. 4). Muchas de estas plantas han sido introducidas en años recientes por viveros comerciales y jardines botánicos. A pesar de que muchas plantas ornamentales permanecen solamente como cultivadas, en Australia, Suiza y algunas regiones de Estados Unidos un alto porcentaje de las especies que son invasoras fueron introducidas deliberadamente como ornamentales (Reichard \& Hamilton 1997, Myers \& Bazely 2003). En Costa Rica, algunas especies introducidas como ornamentales ya se han naturalizado y pueden causar problemas, como es el caso de Spathodea campanulata (Bignoniaceae), cuyas flores producen un néctar tóxico para los visitantes florales, que podría causar daños en la apicultura (Trigo \& dos Santos 2000).

La introducción de plantas ornamentales se ve favorecida por el crecimiento de la industria de la jardinería y por las políticas de globalización (Myers \& Bazely 2003). Costa Rica no es la excepción, pues existen pocas medidas de seguridad establecidas por el gobierno para la importación de especies (Ley $\mathrm{N}^{\circ}$ 7317de Conservación de Vida Silvestre).

Muchas plantas también fueron introducidas como comestibles o industriales y algunas de éstas actualmente son los principales productos agrícolas de exportación del país; tal es el caso del café (Coffea arabica, Rubiaceae) y del banano (Musa x paradisiaca, Musaceae). No obstante, para la gran mayoría de las especies de nuestro catálogo no se pudo averiguar cuál fue el motivo por el que se introdujeron en Costa Rica. Muchas fueron introducidas accidentalmente, porque las semillas llegaron mezcladas con las de otras plantas traídas intencionalmente; esto ha ocurrido también en otros países (Myers \& Bazely 2003).
Un porcentaje importante de las plantas introducidas en Costa Rica tiene su origen en Asia tropical (19.4\%) y en Sudamérica (18.6\%). En ambas regiones las condiciones ambientales son similares a las de Costa Rica, lo que favorece que las especies introducidas sobrevivan y se establezcan. Un alto porcentaje de las especies de origen europeo ya habían llegado antes del siglo veinte y fueron traídas por los españoles durante la época de la Colonia o por algunos gobernantes después de la Independencia de Costa Rica (1821).

Hasta ahora, excepto por muy pocos estudios (Di Stefano et al. 1998, Sierra 2001a,b) y algunas charlas y talleres, en general se ha puesto poca atención a las especies introducidas en Costa Rica. En el caso de las plantas, algunos científicos y personal técnico saben cuáles especies poseen potencial como invasoras, incluso cuáles se encuentran en bosques de áreas protegidas. Sin embargo, esta información no está publicada o no ha sido recopilada adecuadamente; así, es difícil determinar cuáles especies están naturalizadas, qué efectos pueden causar en los ecosistemas naturales y cuáles son las áreas más vulnerables.

Es necesario establecer mejores controles en la introducción de especies, así como incentivar el uso de especies nativas en sectores tales como el forestal, el agropecuario y el de paisajismo urbano. Por último, se espera que esta lista sea una fuente de consulta para todas las personas interesadas en las plantas introducidas, quienes realizan estudios ecológicos, manejan áreas protegidas y protegen cultivos, entre otros.

Agradecimientos. Agradecemos a Carlos O. Morales, Gilbert Barrantes y Jorge Gómez Laurito (Escuela de Biología, Universidad de Costa Rica) por su invaluable ayuda en la revisión de este manuscrito y del catálogo publicado en Internet. Agradecemos a Idea Wild, por su contribución a la realización de este trabajo, y a Andrea Grosse, por su ayuda en la publicación del catálogo en la página web de la Red de Información de Invasoras de IABIN (I3N).

\section{Bibliografía CONSULTADA}

Baker, H.G. 1967. Support for Baker's law - as a rule. Evolution 21: 853-856.

Bernhardt, K.-G. \& M. Koch. 1994. Eingeführte Pflanzen aus Europa als Bestandteil der Vegetation Costa Ricas (Zentralamerika). Bauhinia 11(2): 121-127. Brummitt, R.K. 2001.World Geographical Scheme for Recording Plant Distributions Edition 2. International Working Group on Taxonomic Databases for Plant 
Sciences (TDWG). $137 \mathrm{p}$.

Burger, W. 1977. Flora Costaricensis. Fieldiana, Bot. 35: $1-227$

Burger, W. 1977. Flora Costaricensis. Fieldiana, Bot. 40: $1-291$

Burger, W. 1983. Flora Costaricensis. Fieldiana, Bot., n.ser. 13: 1-255

Burger, W. 1986. Flora Costaricensis. Fieldiana, Bot., n.ser. 18: 1-92

Burger, W. 1991. Flora Costaricensis. Fieldiana, Bot., n.ser. 28: 1-52

D'Antonio, C. \& P. Vitousek. 1992. Biological invasions by exotic grasses, the grass-fire cycle and global change. Ann. Rev. Ecol. Syst. 23: 63-88.

Daehler, C.C. 1998. The taxonomic distribution of the invasive angiosperm plants: ecological insights and comparison to agricultural weeds. Biol. Conserv. 84: 167-180.

Daehler, C.C., J.S. Denslow, S. Ansari \& H-C. Kuo. 2004. A risk-assessment system for screening out invasive pest plants from Hawaii and other Pacific islands. Conserv. Biol. 18(2): 360-368.

Di Stéfano, J.F., L.A. Fournier, J. Carranza, W. Marín \& A. Mora. 1998. Potencial invasor de Syzygium jambos (Myrtaceae) en fragmentos boscosos: El caso de Ciudad Colón, Costa Rica. Rev. Biol. Trop. 46: 567-574.

Espinosa-García, F.J., J.L. Villaseñor \& H. Vibrans. 2004. The rich generally get richer, but there are exceptions: Correlations between species richness of native plant species and alien weeds in Mexico. Diversity Distrib.10: 399-407.

Hammel, B.E., M.H. Grayum, C. Herrera \& N. Zamora (eds). 2003a. Manual de Plantas de Costa Rica. Vol. II. Monogr. Syst. Bot. Missouri Bot. Gard. 92: 1694.

Hammel, B.E., M.H. Grayum, C. Herrera \& N. Zamora (eds). 2003b. Manual de Plantas de Costa Rica. Vol. III. Monogr. Syst. Bot. Missouri Bot. Gard. 93: 1884.

Higgins, S.I., D.M. Richardson, R.M. Cowling \& T.H. Trinder-Smith. 1999. Predicting the landscape-scale distribution of alien plants and their threat to plant diversity. Conserv. Biol. 13(2): 303-313.

Holdridge, L. R., L.J. Poveda \& Q. Jiménez. 1997. Árboles de Costa Rica Vol 1.2 ed. Centro Científico Tropical, San José. 544 p.

Jiménez M., Q., A. Estrada, A. Rodríguez \& P. Arroyo. 1996. Manual dendrológico de Costa Rica. Taller de Publicaciones, Instituto Tecnológico de Costa Rica. Cartago. 165 p.

Judd W.S., C.S. Campbell, E.A. Kellog \& P.F. Stevens.
1999. Plant Systematics: A phylogenetic approach. Sinauer Assoc., Massachusetts. 464 p.

Kollmann, J. \& M. J. Bañuelos. 2004. Latitudinal trends in growth and phenology of the invasive alien plant Impatiens glandulifera (Balsaminaceae). Divers. Distrib. 10: 377-385.

Kühn, I, W. Durka \& S. Klotz. 2004. BiolFlor: a new plant-trait database as a tool for plant invasion ecology. Divers. Distrib. 10: 363-365.

Leon, J. \& L.J. Poveda. 2000. Nombres comunes de las plantas en Costa Rica. Edit. Guayacán, San José $915 \mathrm{p}$.

Mabberley, D.J. 1997. The plant-book. Cambridge Univ. Press, U.K. 858 p.

Maron, J.L. \& M. Vila, 2001. When do herbivores affect plant invasion? Evidence for the natural enemies and biotic resistance hypotheses. Oikos 95: 361-373.

Mauchamp, A. 1997. Threats from alien plants species in the Galápagos Islands. Conserv. Biol. 11(1): 260263.

Myers, J.H. \& D.R. Bazely. 2003. Ecology and control of introduced plants. Cambridge Univ. Press, U.K. $313 \mathrm{p}$.

Parker, I.M., D. Simberloff, W.M. Lonsdale, K. Goodell, M. Wonham, P.M. Kareiva, M.H. Williamson, B von Holle, P.B. Moyle, J.E. Byers \& L. Goldwasser. 1999. Impact: toward a framework for understanding the ecological effects of invaders. Biol. Invasions 1 : 3-19.

Pauchard, A., L.A. Caviares \& R.O. Bustamante. 2004 Comparing alien plant invasions among regions with similar climates: where to from here? Divers. Distrib. 10: 371-375.

Pyšek, P. 1998. Is there a taxonomic pattern to plant invasions? Oikos 82: 282-294.

Pyšek, P., J. Sádlo \& B. Mandák. 2003. Alien flora of the Czech Republic, its composition, structure and history. Plant Invasions: Ecological Threats and Management Solutions. p. 113-130.

Rambuda, T.D. \& S.D. Johnson. 2004. Breeding systems of invasive alien plants in South Africa: does Baker's rule apply? Divers. Distrib. 10: 409-416.

Reichard, S.H. \& C.W. Hamilton. 1997. Predicting invasions of woody plants introduced into North America. Conserv. Biol. 11(1): 193-203.

Rejmanek, M. \& D.M. Richardson. 1996. What attributes make some plant species more invasive? Ecology 77(6): 1655-1661.

Richardson, D.M., P. Pyšek, M. Rejmánek, M.G. Barbour, F.D. Panetta \& C.J. West. 2000 Naturalization and invasion of alien plants: concepts and definitions. Divers. Distrib. 6: 93-107. 
Rivas, M. 1996. Cactáceas y suculentas del Jardín Botánico Lankester. EUNED, San José. 120 p.

Sierra, C. 2001a. El cerdo cimarrón (Sus scrofa, Suidae) en la Isla del Coco, Costa Rica. Composición de su dieta, estado reproductivo y genética. Rev. Biol. Trop. 49: 1147-1157.

Sierra, C. 2001b. El cerdo cimarrón (Sus scrofa, Suidae) en la Isla del Coco, Costa Rica: escarbaduras, alteraciones al suelo y erosión. Rev. Biol. Trop. 49: 1158-117.

Standley, P.C. 1937a. Flora of Costa Rica. Part I. Field Mus. Nat. Hist., Bot. Ser. 18:1-398.

Standley, P.C. 1937b. Flora of Costa Rica. Part II. Field Mus. Nat. Hist., Bot. Ser. 18: 401-780.
Standley, P. C. 1938a. Flora of Costa Rica. Part III. Field Mus. Nat. Hist., Bot. Ser. 18: 783-1133.

Standley, P.C. 1938b. Flora of Costa Rica. Part IV. Field Mus. Nat. Hist., Bot. Ser. 18: 1133 -1571.

Trigo, J.R. \& W.F. dos Santos. 2000. Insect mortality in Spathodea campanulata Beauv. (Bignoniaceae) flowers. Rev. Bras. Biol. 60(3): 537-538.

Vitousek, P.M., C.M. D’Antonio, L.L. Loope \& R. Westbrooks. 1996. Biological Invasions as Global Environmental Change. Amer. Scientist 84: 468-78

Weber, E.F. 1997. The alien flora of Europe: a taxonomic and biogeographic overview. J. Veget. Sci. 8: 565-572.

\section{Ubicación del Catálogo de plantas introducidas en Costa Rica:}

http://i3n.iabin.net/participants/costarica.html

Cuadro 1. Familias de plantas con más de 30 especies introducidas en Costa Rica y, según Mabberley (1997) y Judd et al. (1999), número de especies de estas familias en el mundo.

\begin{tabular}{|c|c|c|}
\hline Familia & $\begin{array}{c}\text { Número de especies } \\
\text { introducidas en Costa Rica }\end{array}$ & $\begin{array}{c}\text { Número de especies en el } \\
\text { mundo }\end{array}$ \\
\hline Poaceae & 118 & 9500 \\
\hline Fabaceae & 74 & 12150 \\
\hline Asteraceae & 49 & 22750 \\
\hline Cactaceae & 36 & 1400 \\
\hline Arecaceae & 33 & 2780 \\
\hline Myrtaceae & 29 & 4620 \\
\hline
\end{tabular}

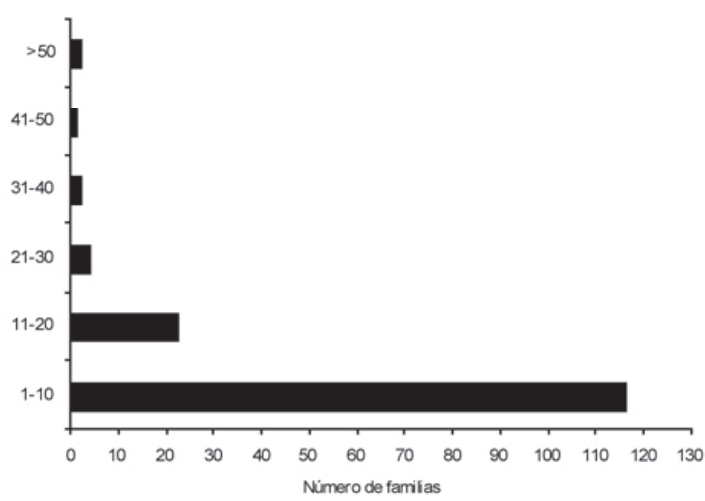

Fig. 2. Número de familias de plantas según el número de especies introducidas en Costa Rica. 


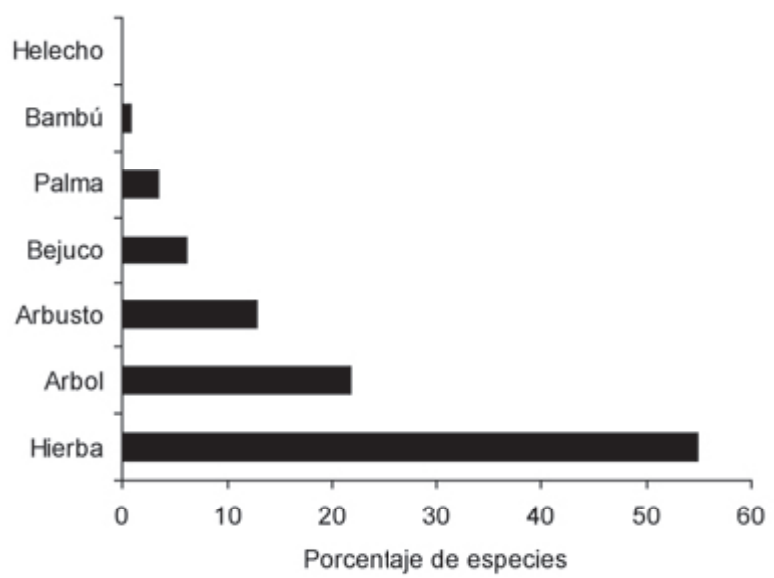

Fig. 3. Porcentaje de especies de plantas introducidas en Costa Rica según su hábito.

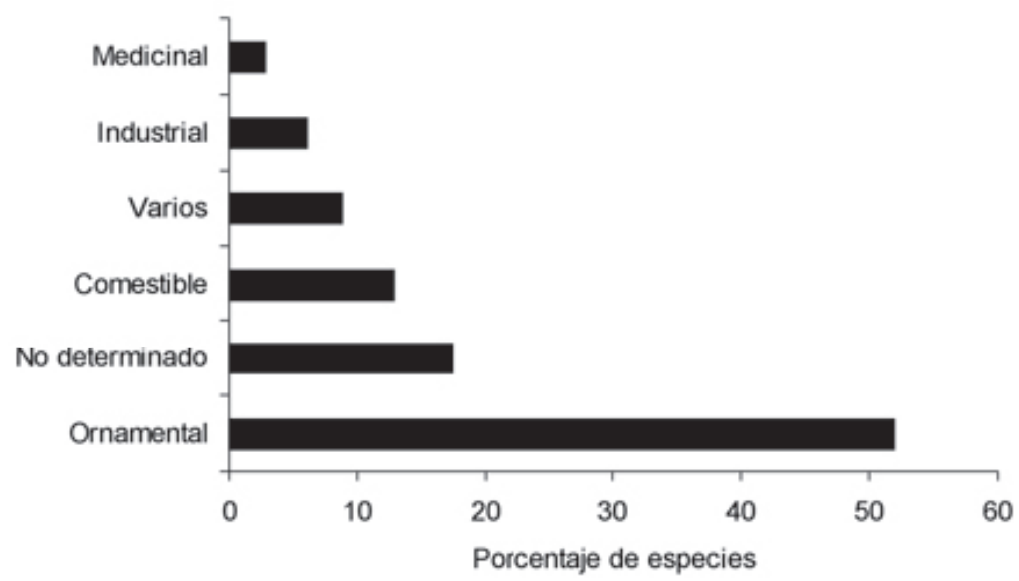

Fig. 4. Porcentaje de especies de plantas introducidas en Costa Rica según su uso.
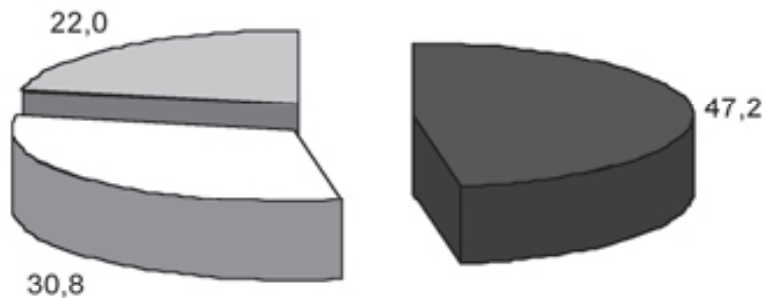

$$
\begin{aligned}
& \text { - Cultivada } \\
& \square \text { No determinado } \\
& \square \text { Naturalizada }
\end{aligned}
$$

Fig. 5. Porcentaje de especies de plantas introducidas en Costa Rica según su estado poblacional. 


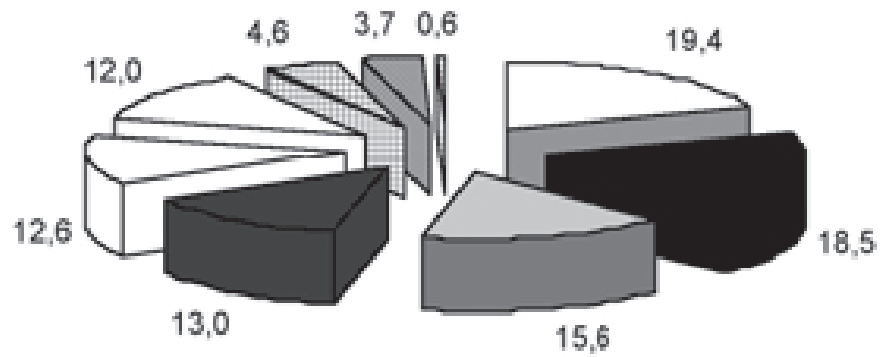

\begin{tabular}{|l|}
\hline - Asia tropical \\
- Sudamérica \\
a Africa \\
a Europa \\
a Norteamérica \\
a Varios \\
a Asia templada \\
a Australasia \\
a Pacirico
\end{tabular}

Fig. 6. Porcentaje de especies de plantas introducidas en Costa Rica según su origen geográfico. 